\title{
Ma che freddo fa? Come si rabbrividisce in Toscana
}

\author{
Matilde Paoli
}

PUBBLICATO: 23 DICEMBRE 2016

\section{Quesito:}

Per salutare i nostri utenti prima del Natale abbiamo pensato di proporre le diverse denominazioni e locuzioni usate in Toscana per indicare il freddo pungente della stagione invernale. Per farlo abbiamo soprattutto interrogato il corpus di ALT-web L'Atlante Lessicale Toscano in rete. Dedichiamo questa scheda in particolare a tutti coloro che per lavoro, perché vittime del terremoto o perché costretti dalla necessità, devono affrontare il gelo anche in questi giorni di festa.

\section{Ma che freddo fa? Come si rabbrividisce in Toscana}

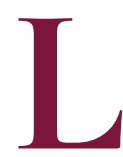

a forma più diffusa in Toscana è zizzola (con tutte le $z$ sonore come in zanzara), che in area nord occidentale e all'Elba può essere anche un verbo (un freddo che si zizzola o si zizzola dal freddo) - o zizzolo a Rio nell'Elba o zizzera in alta Valtiberina e a Pitigliano nell'Amiata - che, se aggiunta a sizza di area fiorentino valdarnese, copre l'intera Toscana linguistica. Occupano complessivamente una vasta area anche strizza (che, specialmente in area lucchese e pisana può essere anche verbo: un freddo che si strizza o che strizza l'ossa), strizzata (di freddo) e strizzo (il tempo è a strizzo o freddo strizzo), diffusi soprattutto nel senese, nel grossetano-massetano, in area fiorentina settentrionale, nel pratese, nel pistoiese e nel pisano settentrionale; strizzone in area fiorentina occidentale.

Striggine (raramente anche il tempo è a striggine o strigginoso) s'incunea nell'area precedente coprendo il fiorentino della Val di Sieve e l'area compresa tra il pisano centro-meridionale il livornese il grossetano settentrionale e il senese occidentale.

Strinore è in area orientale dalla Valtiberina all'Amiata, mentre si strina dal freddo in area occidentale (Garfagnana e Montagna Pistoiese, pisano, Colline Metallifere e Elba e anche in due punti dell'Amiata). In entrambe queste aree troviamo anche stridore, termine che appare registrato dalla I alla IV Crusca ("Diciamo anche Stridore, a Freddo eccessivo") e fu usato nei suoi Saggi di naturali esperienze dal Sollevato ovvero l'accademico conte Lorenzo Magalotti.

Si bubbola dal freddo in area sia fiorentina sia aretina sia senese.

Nella Val di Chiana senese fino all'Amiata settentrionale c'è uzza, uzzetta o uzzolina mentre è bruzza, bruzzina, brunzina o bruggina nell'aretino e ancora nella Val di Chiana.

La léppa è il freddo soprattutto in area pistoiese, ma a Badia Prataglia e a Chiusi della Verna, in Casentino si dice un freddo che leppa (olippa), probabili testimoni della forma piu antica, visto che leppare "Usa[va]si anche dal volgo per Togliere, levar via" come attesta la prima edizione del Vocabolario degli Accademici della Crusca. Si tratta, insomma, di un antico modo per dire, come ancora si dice in Casentino, ma anche altrove, un freddo che porta via.

In Garfagnana c'è il sidro e dal freddo si assidrisce, che sarà affine all'assiderare "Agghiacciare, agghiadare, e quasi morir di freddo" già della seconda Crusca. Sempre in lucchesia, ma anche nel pistoiese il sinibbio, che in Versilia si presenta come zenibbio, è un vento freddo che trascina con sé il nevischio. 
Il sidio è concentrato soprattutto nella Chiana senese. Ed è questa una parola antica perché il sido è "freddo eccessivo" già nella Crusca del I6г2 e anche era nelle quattrocentesche Rime del Burchiello Poeta Fiorentino: "La stella Tramontana, è suta folle, A porsi in luogo da morir di sido".

E poi ancora: bisánfora o disánfora indicano il freddo soltanto a Volterra e a Montecatini Val di Cecina; tirizzánfola e tirizzámpola sono usati rispettivamente a Treppio, nella Montagna pistoiese, e a Scarlino, in Maremma; la sirizzana è in alta Val di Sieve e in Casentino.

Soltanto la Lunigiana conosce ferdura, fardura, freddura, mentre la ghiacciura è a Porto Santo Stefano, nell'Argentario, e a Capoliveri, nell'Isola d'Elba.

Infine solo a Olmo, nei pressi di Arezzo, e a Pontremoli, in Lunigiana, si usa brusco riferito al tempo freddo cosi come si usava già nella trecentesca Cronica del Villani, ci dice la terza Crusca, "Onde Rabbruscarsi il tempo, diciamo, Quando si turba, e raffredda".

Il freddo può far tante cose: il freddo stricca, cioè comprime come in una morsa, frizza, il freddo arrabbia e morde; oppure infila o entra nell'ossa, ma anche le buca o le strizza. E poi c'e il freddo che bucchia, pela, sbuccia, spella o monda e il freddo che porta via, che punge, schianta o sghiaina o fa rimpelluccire.

Dal freddo si gela, si móre, s'abbaia, s'arrocchetta, si grenna, si zilla, si rassega, si pipa. E si batton le gazzette, anche a Firenze.

Ma ancora non è finita perché c'è anche un freddo bestia, biscia, rospo; un freddo boia, mostro; un freddo buggerone, buscherone, bussone. Il freddo può esser crudo, lecchino, marmato, pizzichino, rio, salvatico, sodo.

Naturalmente c'è il freddo da lupi e quello da cani e il freddo cane. Parlando di cani si dice quando è freddo che passano i cani senza coda; anche perché il freddo porta via la coda ai cani. Gli uccelli, poi, volan o passan bassi, tanto bassi che si piglia gl'uccelli con le mani.

Per concludere ci piacerebbe proprio tanto che fosse vero, alla lettera, il proverbio antico, anche di Crusca, Dio manda il freddo secondo i panni.

\section{http://www.vocabolariofiorentino.it/}
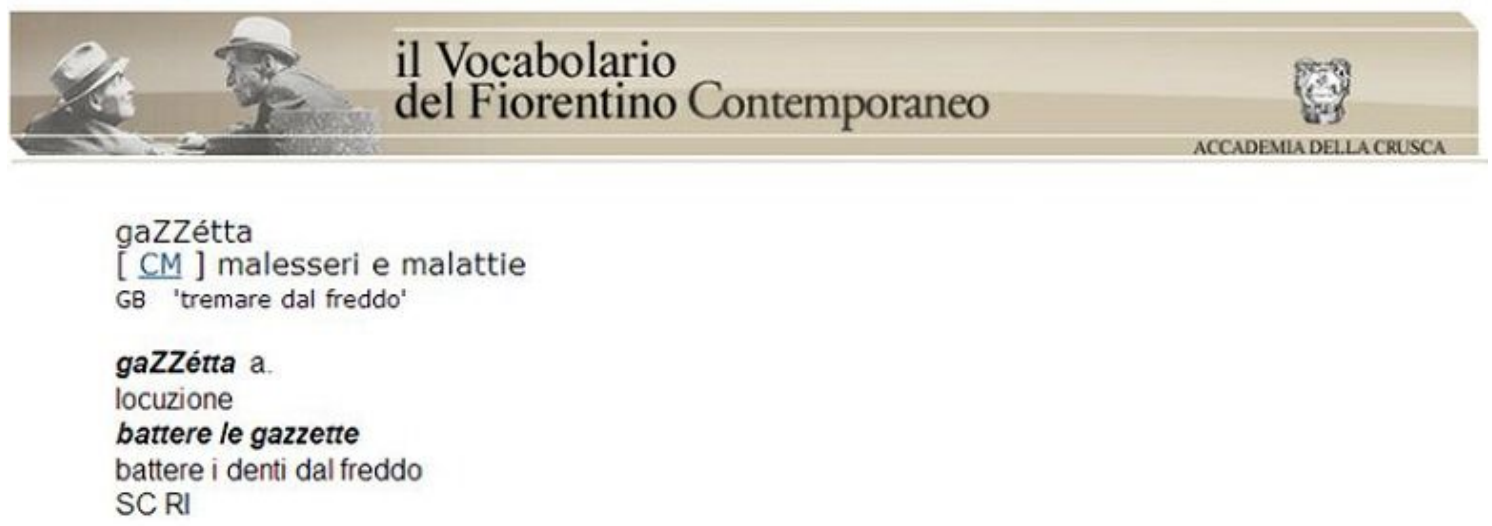

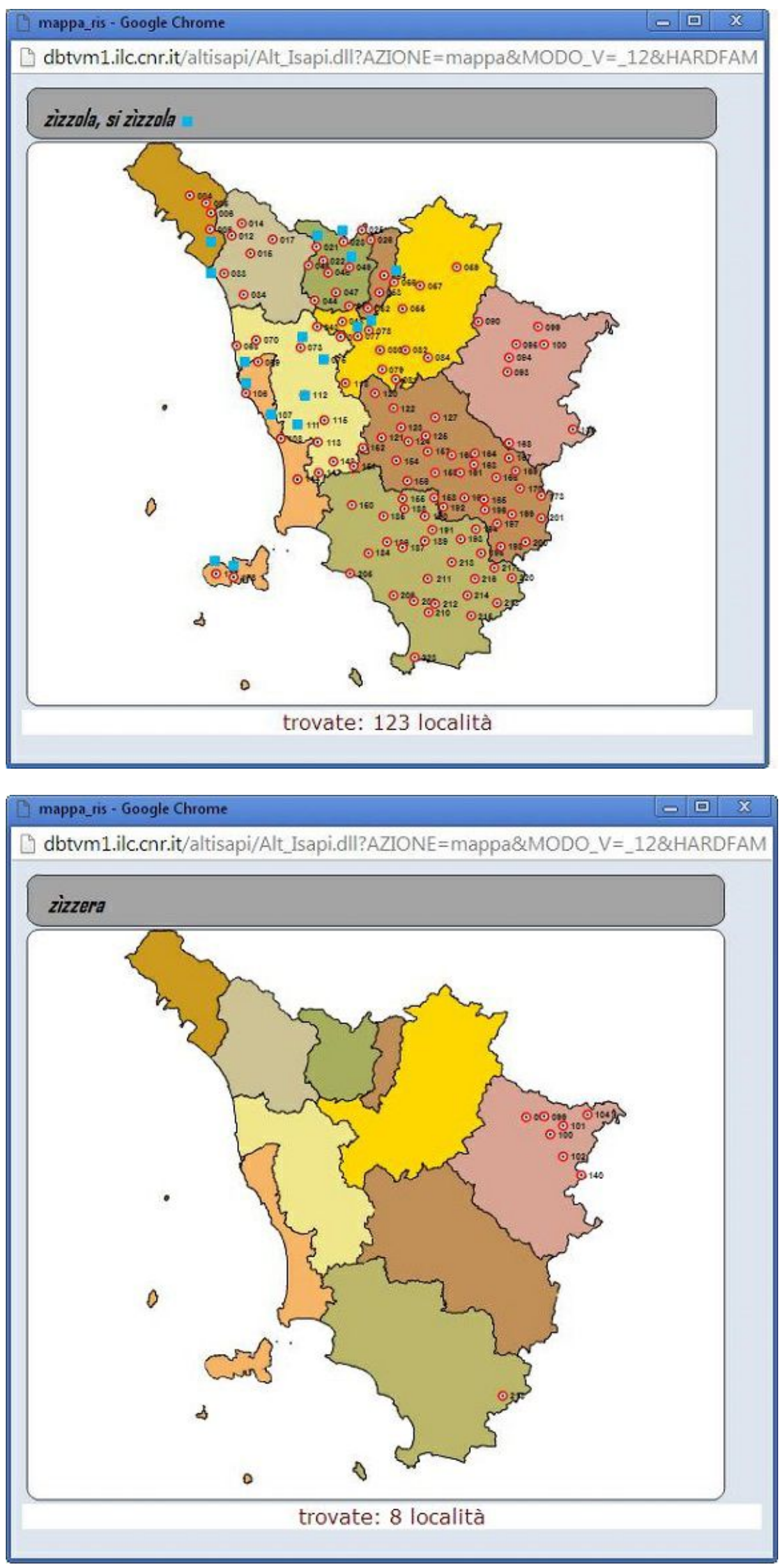

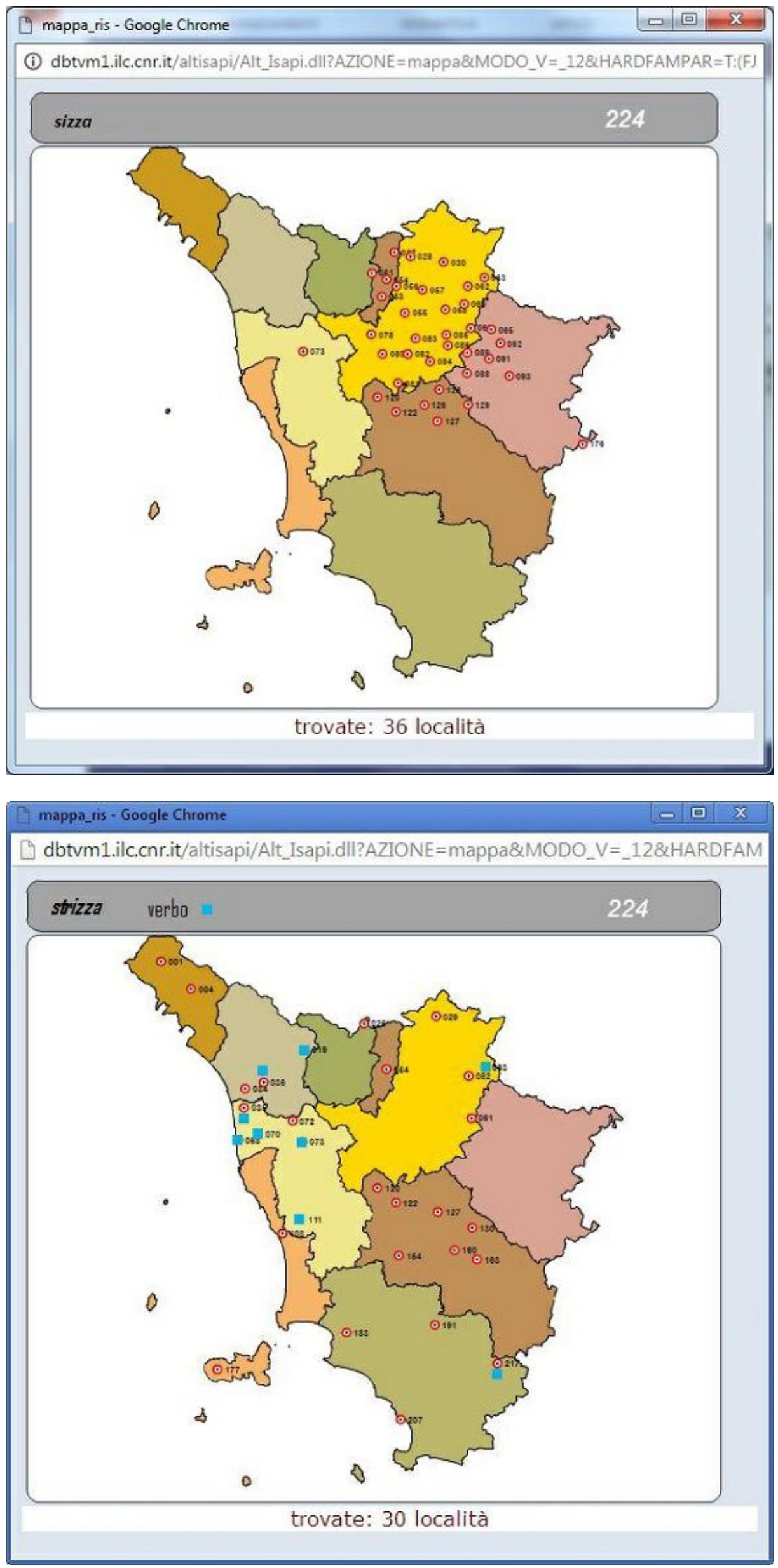

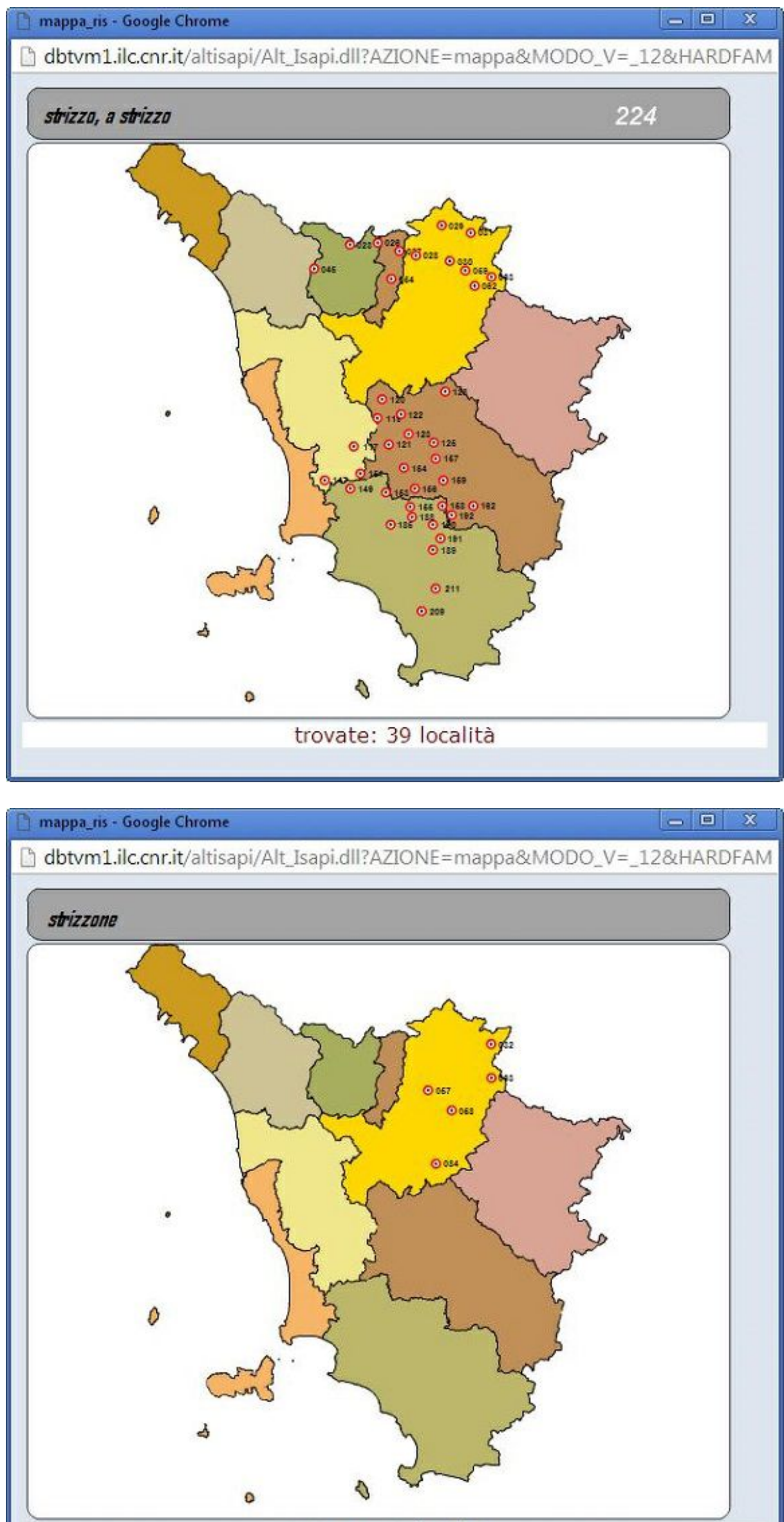

trovate: 5 località 

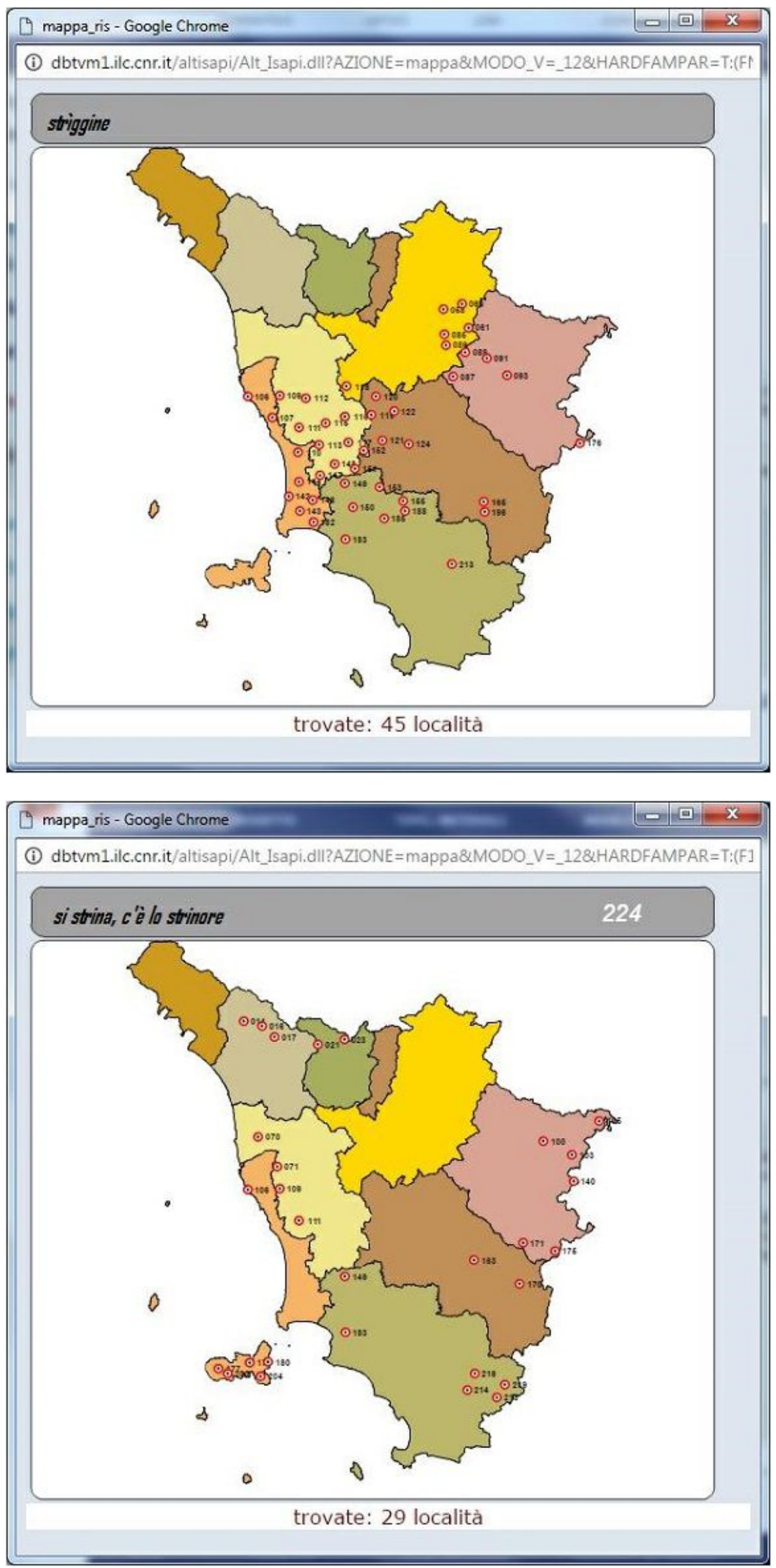

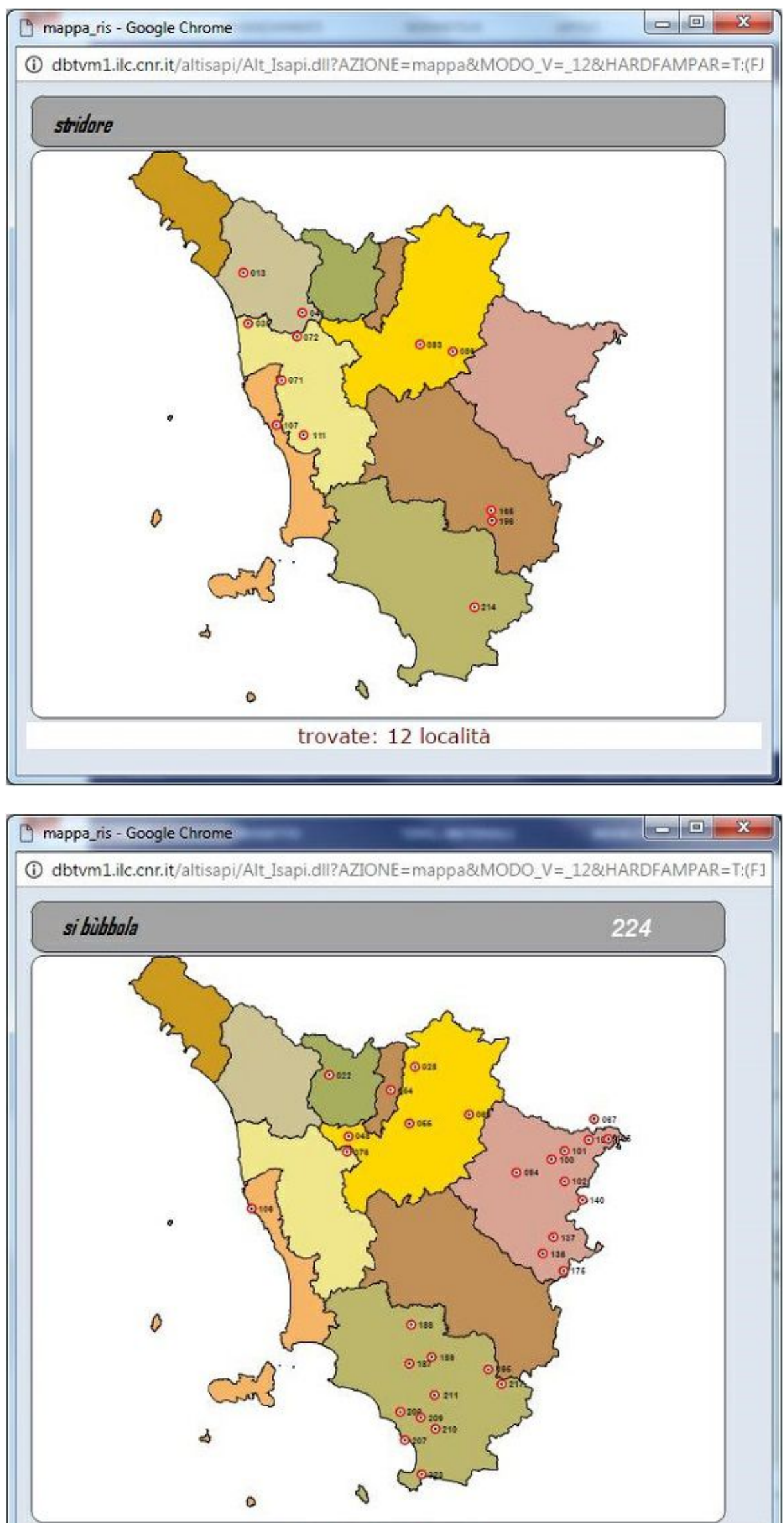

trovate: 30 località 

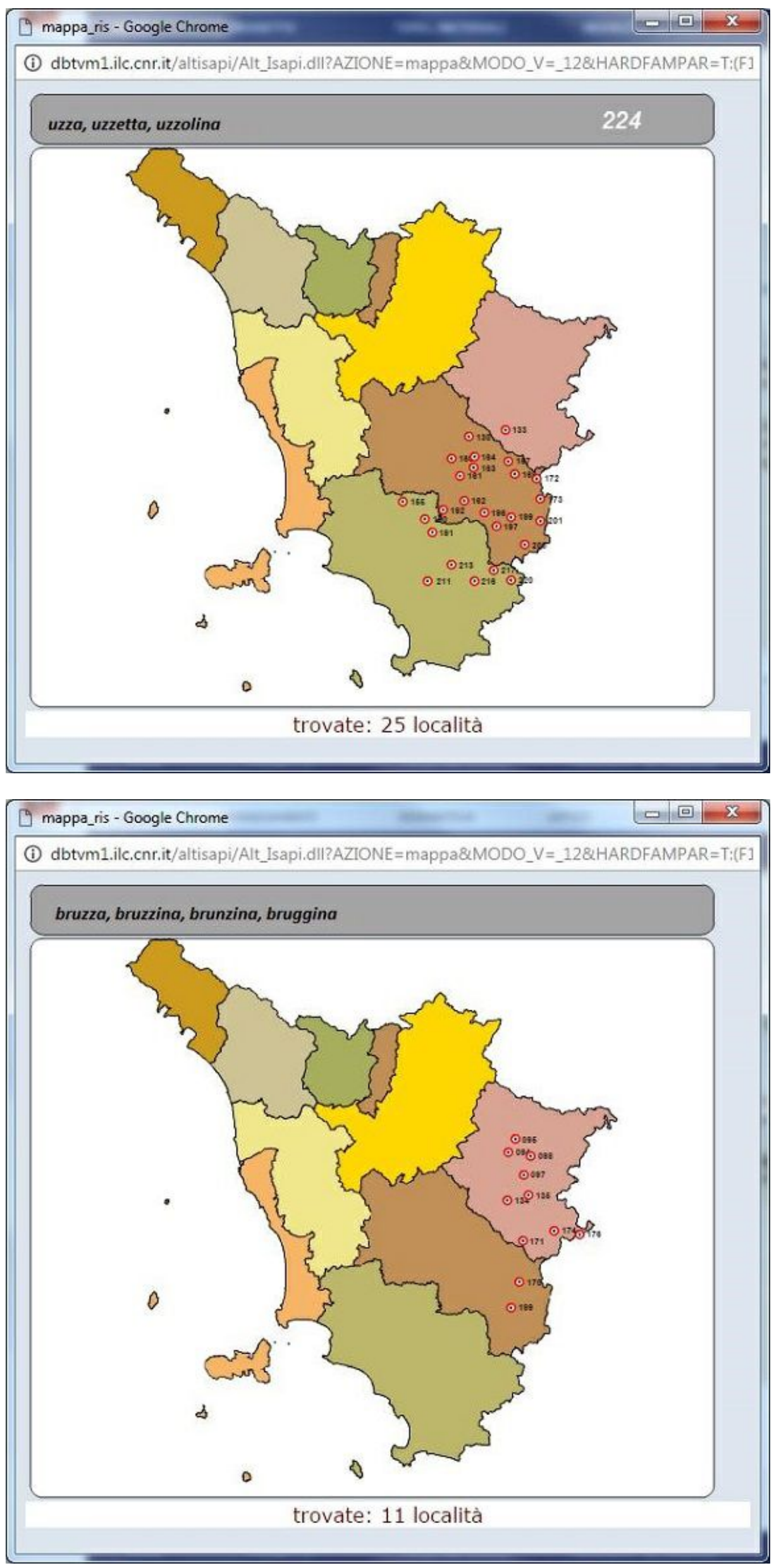

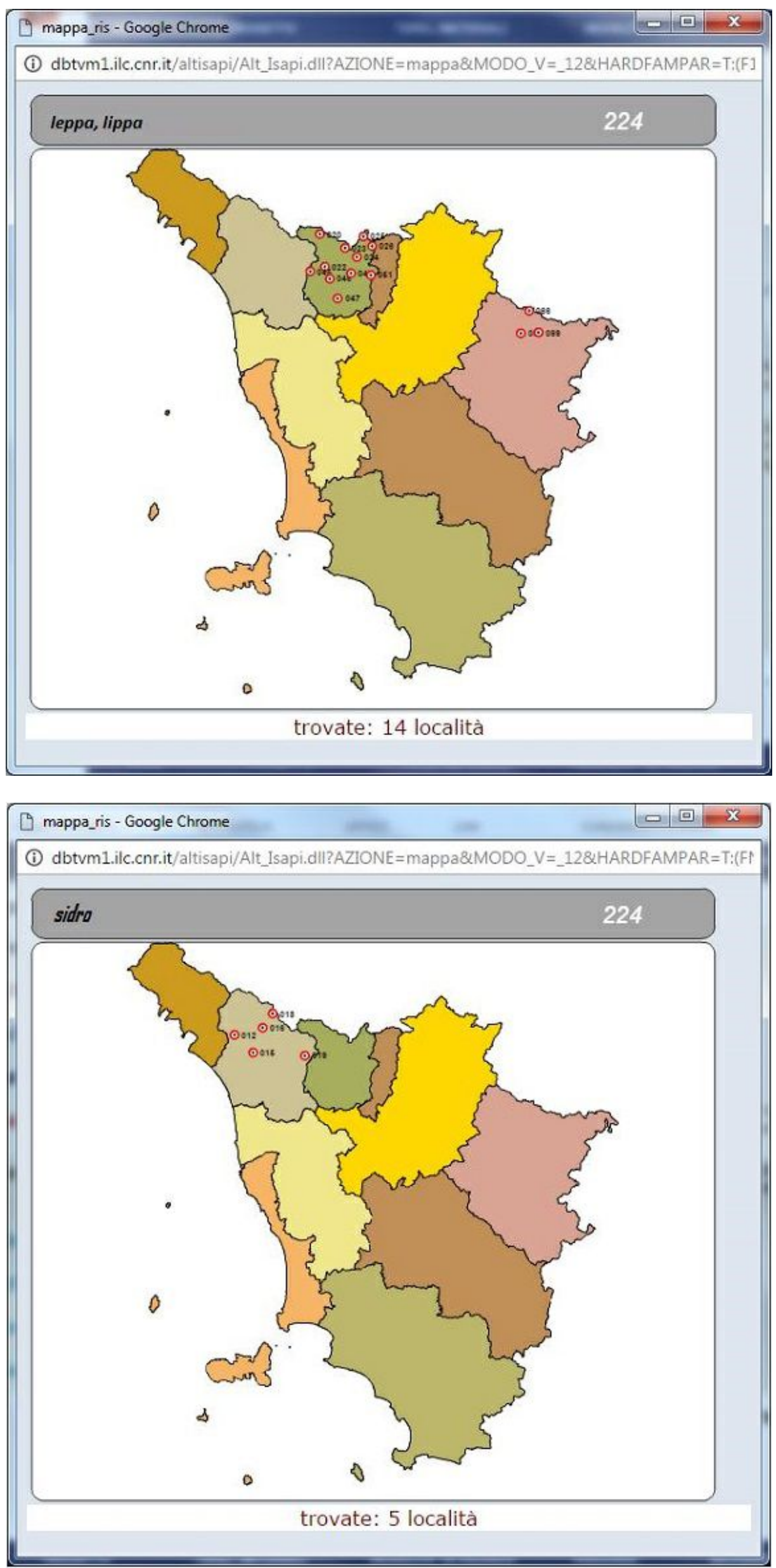

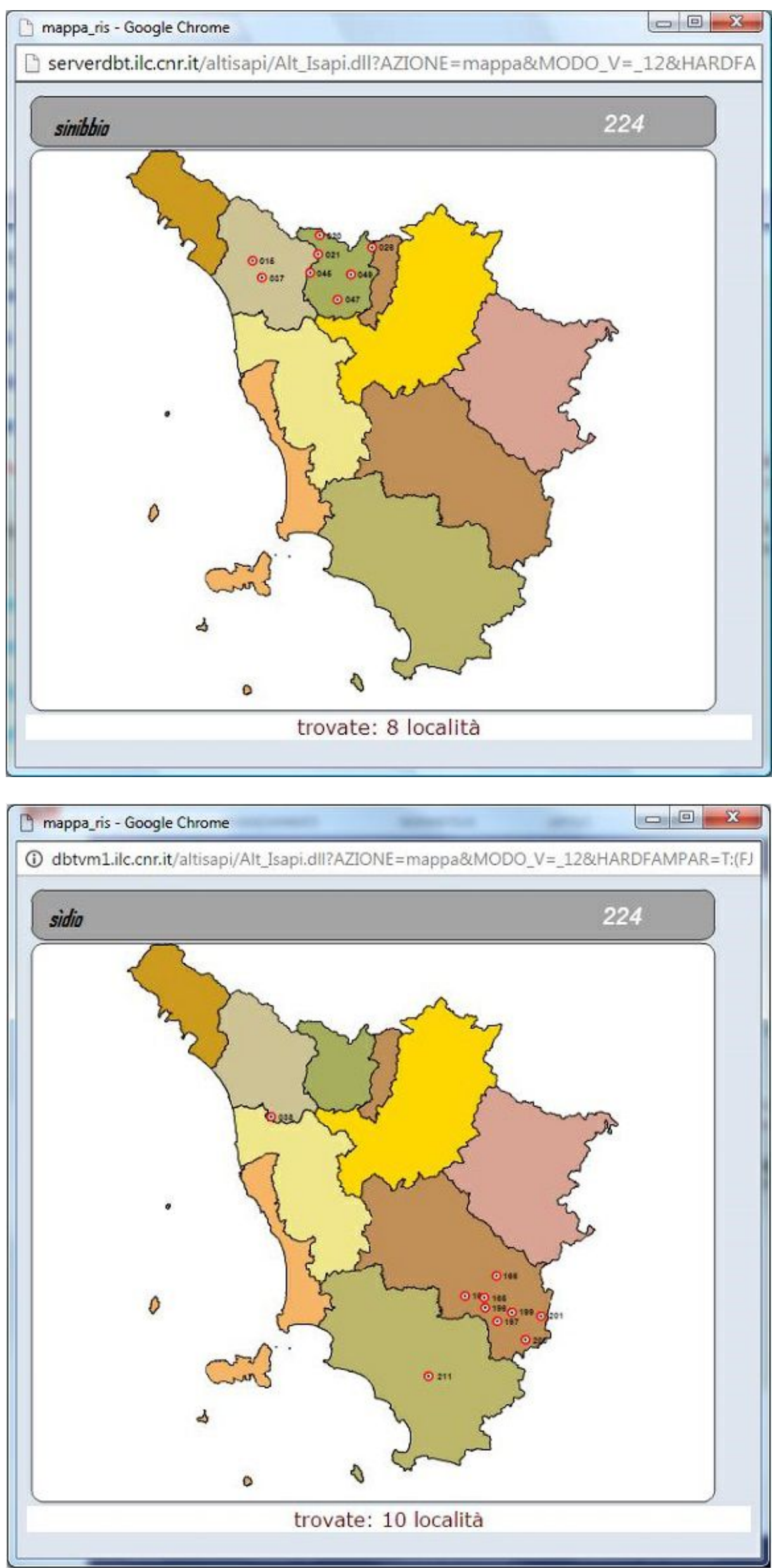

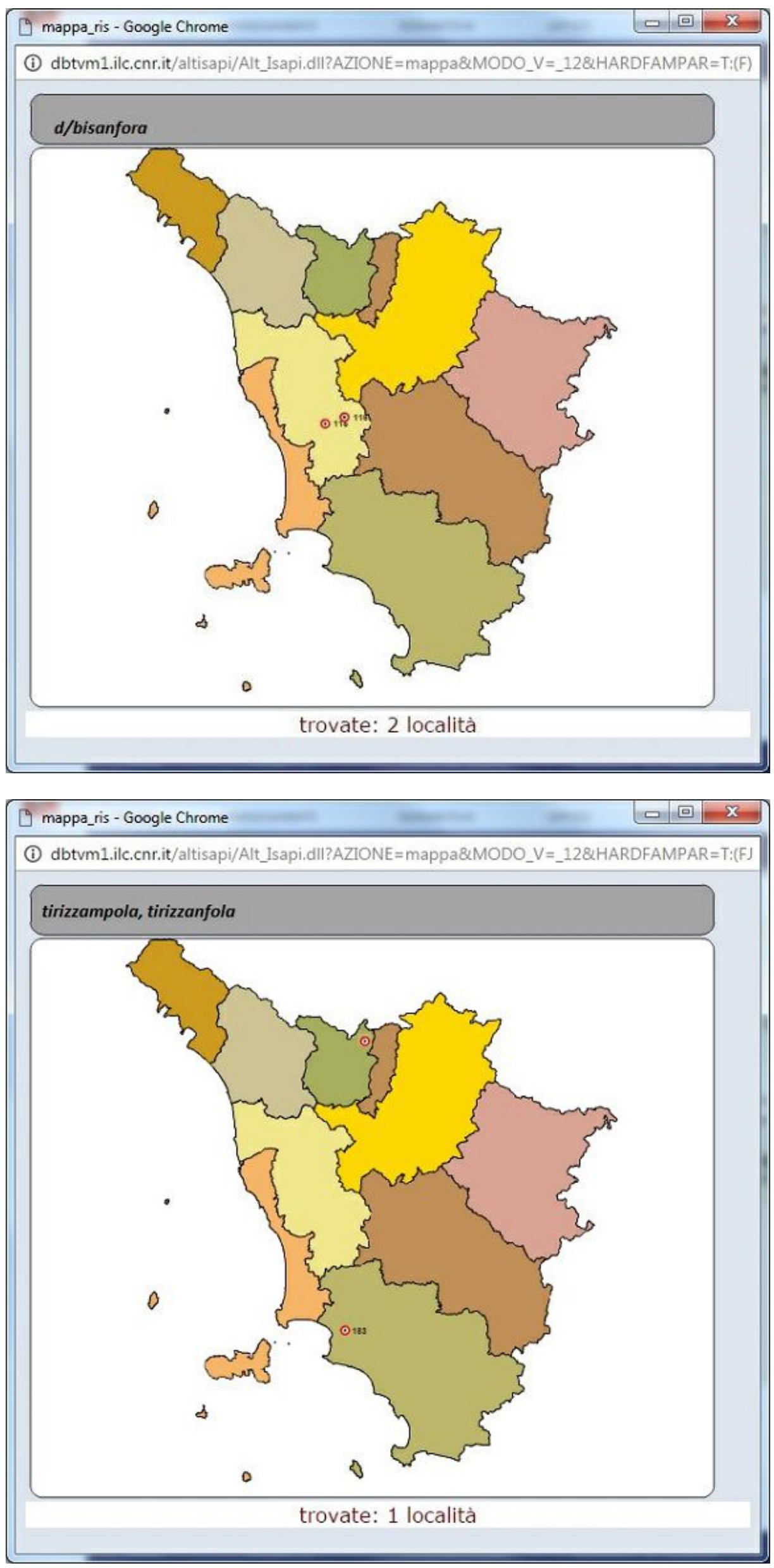

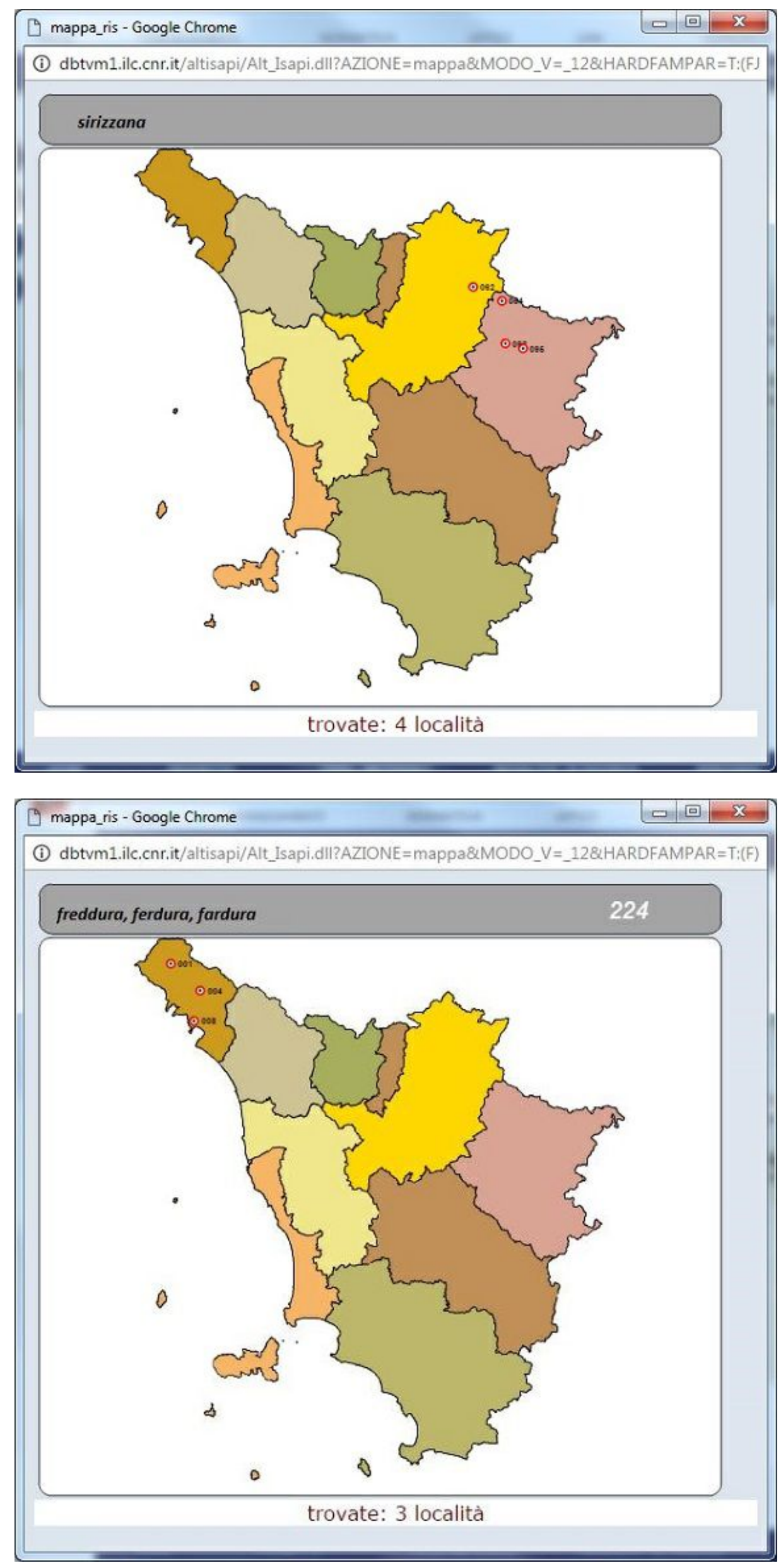


\section{Cita come:}

Matilde Paoli, Ma che freddo fa? Come si rabbrividisce in Toscana, "Italiano digitale", 2016, XI, 2019/4 (ottobre-dicembre)

DOI: 10.35948/2532-9006/2020.3264

Copyright 2016 Accademia della Crusca

Pubblicato con licenza creative commons CC BY-NC-ND 